\title{
Analysis of Science Textbook Pictures about 'Energy' and Pupils' Readings of Them
}

Fani Stylianidou, Institute of Education, University of Sussex, United Kingdom Fiona Ormerod, University of Lancaster, United Kingdom

Jon Ogborn, Institute of Education, University of Sussex, United Kingdom

Correspondence: $\quad$ Fani Stylianidou

Science and Technology Group

Institute of Education

20 Bedford Way

London WC1H 0AL

Tel: $\quad 02076126878$

Fax: 02076126792

e-mail: f.stylianidou@ioe.ac.uk 


\title{
Analysis Of Science Textbook Pictures About 'Energy' And Pupils' Readings Of Them
}

\begin{abstract}
This article outlines the findings of the part of the 'Science Teacher Training in an Information Society' (STTIS) project concerned with describing the possible difficulties the pupils have when 'reading' science textbook pictures about 'energy'. Six documents were selected on the basis that they had some of the textual/graphical features previously identified by the project as potentially presenting difficulties to pupils. The pupils' readings of these were investigated using a questionnaire and a follow-up interview. The analysis of three of the documents and of twelve pupils' readings of them is reported in this paper. The results confirm the hypothesis that the 'reading' of science textbook pictures is not at all trivial for pupils and conclude that teachers need to spend time and effort talking through the meaning of the images with them. They also suggest that the list of textual/graphical features used in this research is a good starting point for this kind of critical examination.
\end{abstract}

\section{Introduction}

As part of the research conducted for STTIS, we undertook to investigate the possible difficulties pupils face when reading science textbook pictures about 'energy' [see introduction of the mini-issue].

The fact that 'energy' is deemed one of the most important and yet one of the most difficult topics of secondary school science made the topic an excellent candidate for a project which aims to produce findings which are valid across different countries. Secondly, although many research findings already exist on the difficulties the pupils have with the ideas involved, there have been considerably fewer studies of difficulties with representations of these ideas.

The common research framework within which we operated identified as a general concern the fact that pupils necessarily interpret information through their own conceptions - their 'theoretical lenses'. A provisional list of textual/graphical features of images, which may present difficulties for students in reading and/or interpreting documents* containing images, was generated based on previous investigations and study of the relevant research. Those relevant to our investigation are:

- Images requiring interpretation of the roles of elements representing both real world and schematic or symbolic entities. $(R / S)$

- Images whose interpretation requires certain elements to be given importance or be highlighted, often in relation to textual/graphical features which makes them salient, or do not make them salient. (SEL)

- Images containing elements which require appropriate readings of symbols, and which contain examples of synonymy, homonymy and/or polysemy of symbols. (SIM)

- Documents with images requiring verbal elements to be read as an important part of the whole, including verbal elements included within the image or used as captions. (VE)

- Documents containing more than one image requiring interpretation of relationships among the different images. (INT)

\footnotetext{
* The word 'document' is used in this paper to refer to the combination of text and images on a page.
} 
- Images which make important use of compositional structures requiring the reading of spatial distributions and different representational structures. (CS)

Our understanding for the last category is based on Kress and Van Leeuwen's 'Reading Images: The Grammar of Visual Design' (1996). According to them there are two types of representational structure, the narrative and the conceptual, as seen in figure 1:

Figure 1. Main types of visual representational structure

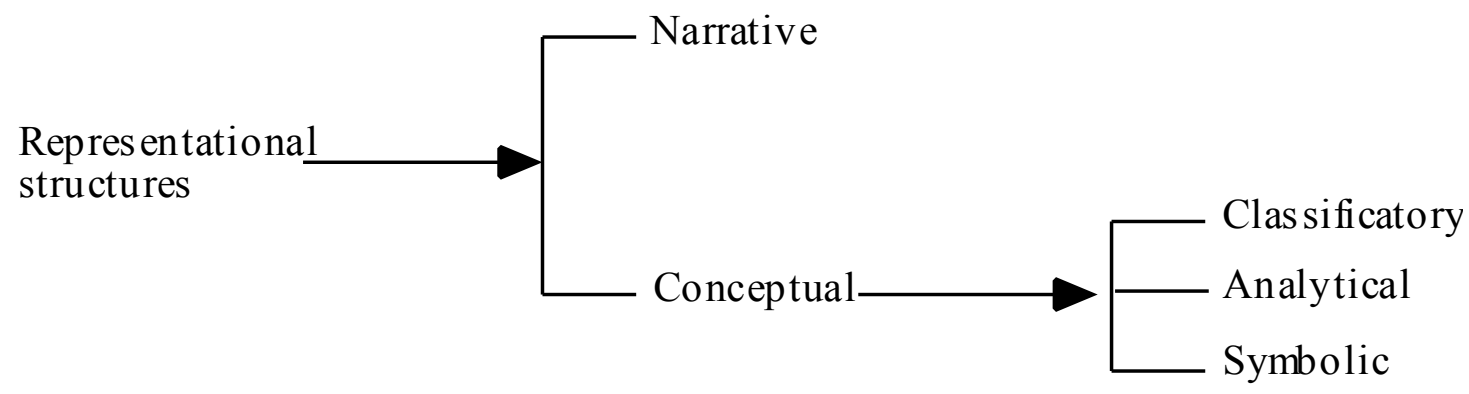

- the narrative representation depicts some kind of transaction between participants in a transitory relationship; it can be either naturalistic (e.g. painting of action at a particular moment) or abstract (e.g. lines indicating weather patterns on a particular day);

- the conceptual representation depicts permanent, fixed relationships and characteristics: showing how things can be categorised, or the characteristics they have; this too can be either naturalistic (e.g. a realistic drawing of an object which draws attention to its features) or more abstract (e.g. a diagram indicating how an electrical circuit works).

Narrative and conceptual representations may be mixed.

The conceptual representations are of three main kinds:

- 'Classificational processes relate participants to each other in terms of a "kind of" relation, a taxonomy'(p81). Examples include diagrammatic tree structures depicting the hierarchical structure of an organization.

- 'Analytical processes relate participants in terms of a part-whole structure'(p89). Examples include maps, or the schematic drawing of a car engine.

- 'Symbolic processes are about what a participant means or is'(p108). Examples include expressionist landscapes and abstract representations.

In the analysis of the textbook pictures we will be making use of these ideas and terms.

\section{Data collection and analysis}

For our investigation six documents containing pictures about 'energy' were selected from a collection of 46 science textbooks published in the years 1966-1997. One of the criteria for the choice of these documents was that they belonged to popular science textbooks with a strong commitment to visual communication of scientific ideas. Another criterion was that they had some of the features identified in the above list, and thus were open to the danger of creating difficulties to the pupils-readers.

We also took care that they had different representational structures (in the sense outlined above), so that we could investigate the effects of these different structures in the meanings the pupils draw from a document. As a result, we ended up with three documents of predominantly narrative structure, and three of predominantly conceptual structure. Of the 
first kind of documents one makes use of naturalistic pictures, and the other two of a combination of schematic and symbolic ones. Similarly, in one of the second kind of documents the representations are naturalistic, whereas in the other two the representations are more abstract. Also, of the latter documents, one focuses mainly on classificational and analytical processes and the other on symbolic processes.

Since the documents were taken from existing texts, unlike those examined in the paper by Ametller and Pintó, they promote a variety of perspectives on energy, including both 'transfer' and 'transform' points of view. For the purpose of this research, we did not take a position regarding which perspective to favour. Our main concern here was with the nature and interaction of graphic elements.

The documents were first analysed within the above framework and a questionnaire was designed for each of them based on this analysis. It was a feature of the design that the wording and style of the six questionnaires were very similar, and where appropriate identical. They prompted the pupils to look at the relevant documents and answer questions such as:

- What do you notice first about this/these picture(s)?

- Every picture tells a story. What is the story behind this/these picture(s)?

- What do you have to do or think about to make sense of this/these picture(s)?

- What do you find difficult to make sense of in this/these picture(s)?

Two sets of three documents each together with their corresponding questionnaires were distributed to a total of 104 pupils - each pupil received only one set (of three documents). Twenty of these pupils were subsequently interviewed on their answers to the questionnaires. After an initial examination of the data, 24 pupils' questionnaires (twelve from each set) were selected for thorough analysis. Interview data existed for most of the selected pupils.

This paper will be concerned with the analysis of three of these textbook documents and of the pupils' readings of them. Each document will be examined in turn.

\section{Document 'Energy'}

\section{About the document 'Energy'}

This document is found in the first page of the chapter on 'Energy' in the Nuffield (1987) science textbook for 12-year-old pupils. It consists of a picture, which covers approximately three quarters of the page and some text (see Appendix1).

The picture on the whole is very 'busy' and 'crowded' and conveys activity and movement. It can be described as consisting of many smaller images put on a continuous background, like a 'collage'. These smaller images are the realistic drawings of eleven different means of transport. All of them appear in three-dimensional perspective, the perspective being chosen to highlight (make salient) the motion (with the exception maybe of the train). Thus, all of them give the impression to be on the move heading in different directions and mostly out of the page towards the reader. They look like vectors scattered erratically in space.

Moreover, the train in 2D crosses the page from left to right at the middle of the page, thus splitting the picture in two parts. The upper part is smaller, lies on the right of the page, and is more 'crowded'. The forms of transport depicted there are those of air and water. The bottom part is bigger, covers the bottom half of the page and is less 'crowded'. The means of transport in it are those that travel on the ground; perhaps they are the more real ones compared to the ideal ones in the top part. The earth-water-air organization of the elements in the picture is worth noticing. In addition, the picture attempts to evoke the notion of depth by showing the objects increasing in size from the top to the bottom of the picture, and thus 
the objects in the upper part look as being further away from the reader than the ones in the bottom part. Having said this, all the objects are drawn in roughly the same amount of detail, which also suggests that they are intended to be seen as equally salient.

Without looking at the text, it is hard to guess what is the common feature all these objects have, apart from being 'moving carriers'. The fact that they are all on the move becomes particularly salient when one attempts to make connections between the picture and the title of the chapter - 'Energy' - which features in big bold letters on top. The designers obviously knew that for many children energy is equated with movement and action. Watts (1983) reports that in this case, energy is identified only when there is an outward display of activity; movement of any kind is often given as a reason for energy being involved. How is energy however involved in this observed movement of the depicted objects? The picture does not help in answering this question. It is only by looking at the text that one finds explicitly what the authors intended the pupils to think. In particular, it is the last question in the text that betrays the relevance of the picture to the title of the chapter. The text starts by explaining what 'a technological society' is and then with the aid of the picture attempts to define the notion of 'technological forms of transport'. After asking the children to count the different forms of transport depicted in the picture and to think of any others not depicted, comes the question: 'Can you work out what all these forms of transport need to make them go?' This question points, according to the associated teacher's notes, to the key-idea intended to be conveyed by the document, i.e. the idea that energy is needed to 'make things go'.

Using Kress and Van Leeuwen's framework, we would suggest that, the picture as a whole can best be described as a naturalistic conceptual structure, in that realistic drawings are used to present information about a number of things which exist in a permanent categorical relationship to each other: they are all forms of transport and they all use energy; the intended message is conceptual.

\section{Research questions}

The research questions for this document are:

- Given the observation that the document seems to capitalise on, if not to reinforce, the fact that energy is strongly associated by children with visible motion, how do the pupils 'work out what all these forms of transport need to make them go'?

- How will the pupils respond to the naturalistic conceptual structure of the document? 'Realistic' features (such as perspective, detail, people in action) are often associated with narrative structures, while conceptual representations often take a more abstract form. Here, a large number of representative items (as in an abstract structure) is presented 'realistically' in a semi-naturalistic setting (as in a narrative structure). Does this give rise to any difficulties in the reading of the document?

\section{Pupils' difficulties with the document 'Energy'}

Given the above research questions the categories which proved pertinent in analysing the data collected from the pupils in relation to this document are:

(i) Selecting, highlighting or missing elements in a representation (SEL)

(ii) Mixing narrative versus conceptual visual structure (CS)

(iii) Reading images through theoretical 'lenses'.

(i) Selecting, highlighting or missing elements in a representation (SEL) Selection and highlighting 
While some of the children, such as Rita, enjoy the complexity of the picture:

'It is very lively and busy and interesting to look at..., it's quite cartoony as well so it's nice to study all the details in it'.

others indicate that the number of elements in the small space, and lack of highlighting of specific elements, make the picture difficult to understand - it is hard for them to work out which elements are intended to carry the most meaning. Farida, for example, explains:

'The picture is quite confusing because all the cars and buses and trains and others are all muddled up.

I find it quite difficult because all the pictures are in the same side on top of the other'.

This confusion is dealt with in different ways. Some children, like Farida, feel that the picture is difficult, but that it rewards close detailed observation, focusing on individual elements. She says, 'It did look complicated at first but then I looked at it in detail and it was okay'.

Some point out that it is important to try to see connections between the different components; Felicity, for example, suggests 'Just look at them and try to link everything up'. (The various ways in which different children attempt to do this are discussed further below.)

\section{Missing information}

The images are not simply intended to be understood as representations of modes of transport, but to carry another meaning, beyond this, about different forms of technology and related energy transfer and use. However, this meaning is not made explicit; the use of energy is only implied. Predictably, not all the children grasp the full intended meaning, as the idea of 'transport' overrides other possible interpretations.

Most of the children mention transport. Some focus very generally on what they see; for example, Jimmy says 'This is a good picture - I thought we were talking about transport', and Rita thinks 'It's obvious what it's about: Transport'. Some children focus on particular aspects of the vehicles; for example, Samina notes specific body parts: 'nearly all of the things have wheels on it'; and several children, such as Raymond, focus on the representation of speed: 'a lot of vehicles, and they all seem to be moving fast'.

The majority do not move beyond the images of vehicles. Farida, however, appears to sense that the picture is intended to tell her more, but that it is not communicating fully its intended message. She says it is 'too difficult - I don't understand any of it'; the problem, as she sees it, is that the picture simply shows which kinds of transport we can use. 'Cos there's nothing else there'.

Only around half the children see the vehicles as a starting point for thinking about energy (a few comment on the helpfulness of the title and written paragraph in leading them in this direction). Several children realise that the picture is about energy in connection with transport, but feel confused as to what they are meant to understand, because of the lack of explicit information. Three children point out that it would be helpful if labels were provided, explaining what the things are, what they are for, and how they work, or as Louise puts it 'how they work, the energy in them, how the energy works'. Left without any helpful signposts, these children have to make sense of what the picture tells them about energy by drawing on their own scientific knowledge.

\section{(ii) Mixing narrative and conceptual visual structures (CS)}

\section{A primarily 'conceptual' approach}

Most of the children do appear to understand the picture primarily as a conceptual structure, representing a particular category of related things: different forms of transport, or different examples of energy use. 
Two children take their analyses slightly further. Rita thinks it is about different forms of transport and how transport is developed over the years, and Felicity interprets the vertical arrangement of the vehicles as representing hierarchical sub-categories of transport types: 'it goes from top to bottom in rank of the fastest to the slowest and the heights they can go'. Since the intended purpose of the picture is not to convey information about height, speed, or historical development, these interpretations may be distracting, rather than helpful.

Raymond takes a conceptual approach but expresses concern about the size and position of objects in the picture, indicating that the 'realistic' characteristics of the images and layout is in a way misleading, since in real-1ife these things are not all seen at once in a small space. He points out 'you have to realise that this isn't the usual size and the way they are usually placed in the city'. $\mathrm{He}$ is drawing attention to a contradiction between the realistic and less realistic features of this representation, which distract from the communication of conceptual meaning.

\section{A mixed 'narrative- conceptual' approach}

A few children appear to interpret the picture as carrying both narrative and conceptual meaning. As a whole it is seen as conceptual (about transport) but individual sections are seen as narrative (about particular events).

Yusuf interprets the bottom section of the picture as carrying narrative meaning: 'people late for work, using more petrol, wasting more energy, and travelling very fast' and some of the vehicles 'stuck in a traffic jam and wasting fuel'. His comments on energy seem to relate directly to the use of fuel in this particular situation. It seems that size and perspective are leading him to interpret this section as 'new', 'foregrounded' information, thus narrowing his focus. At the same time, his criticism of the picture, 'it only shows transport of traveling in road or flying, it doesn't show people walking', indicates a conceptual interpretation: it depicts different forms of transport, but does not, in his view, contain enough information to provide a complete picture of what transport means.

Similarly, Samina interprets the top right part of the picture as a narrative about a helicopter rescuing people and an 'airplane which has just flown up in the air'. She experiences problems with some of the layout, explaining 'What I find difficult is why is the rocket right next to the boat', and expresses concern about the close proximity of electricity and water in the picture, which she knows can be dangerous. Here, the realistic characteristics of the picture are leading her inwards, to the specifics of a particular moment, rather than towards more general concepts. However, she also makes a number of general statements, pointing to commonalities between the objects depicted, indicating that they represent a category of things: types of transport. She focuses on physical structure: 'nearly all of the things have wheels on it'; on how much they are used: 'many people use nearly everything'; on where they are used: 'in the UK' and 'all around the world'; on how they are made: 'people make them using technology'; and their effect on the environment: 'all the things... cause a lot of pollution'. She is clearly trying to extract as much meaning as possible, both narrative and conceptual, from the images, but this does not lead her to understand the picture to be about 'energy'.

All these responses in this section suggest that the depiction of a large number of representative 'types' of object, within a 'semi-naturalistic' setting, in this document, appears to lead to difficulties in understanding the relevance of the individual components and how these are intended to work together to convey meaning.

\section{(iii) Reading images through theoretical 'lenses'}


Those children who actually comment on energy (only just over half the group) talk about it in a variety of ways, revealing different understandings. Some of these understandings are not surprising, others are less predictable.

Energy is associated (as predicted) with action and movement; energy can be fast or slow; humans use energy to walk (although this is not depicted); energy is associated with different forms of transport: vehicles contain, use and make energy; fuels are seen to provide or trigger energy; different things possess different types of energy; and fuel and energy can be wasted, through speed, or other forms of excessive use. Energy is also associated, for two pupils, with electricity; and for one, water and electricity together are associated with electrocution.

These ideas indicate that the children are approaching the document from a range of starting points, some of which may be more useful than others.

\section{Summary}

The children's responses indicate that problems associated with this document seem to stem:

- firstly, from the crowded layout, lack of highlighting, and a mixture of representational modes, which lead to ambiguity of focus and conflicting narrative and conceptual interpretations; and

- secondly, from the lack of explicit verbal or visual information about energy, which leads them to miss the main message about energy all together, or to have to rely on their own prior knowledge in trying to make sense of what it tells them about this.

\section{Document 'Hot on the trail'}

\section{About the document 'Hot on the trail'}

This is a single picture covering almost all of the top-half of the page of a science textbook (Dobson 1987a) for 13 year-old pupils. The heading 'Hot on the trail' seem to imply that something is hidden and on the move and one should look hard to trace it. This 'something', as this section is in the chapter about 'energy', can be assumed to be 'energy'. Indeed the first sentence which introduces the picture reads: 'Tracing energy through a complicated system can be quite interesting'. We would suggest that this sentence serves many functions:

- to remind the reader what the entity s/he should be looking for is;

- to caution the reader that the picture $\mathrm{s} / \mathrm{he}$ is looking at is a complicated one;

- to tell the reader what sort of (mental) activity s/he is expected to be engaged with when reading this picture;

- to dispose the reader positively towards the activity by suggesting that $\mathrm{s} / \mathrm{he}$ is going to find it 'quite interesting'.

Let's now look at the picture (see Appendix 2). At first sight one takes it to depict the water cycle. It is basically a narrative image (with analytic aspects) which holds the conceptual structure of a cycle. The image works on two levels: on the one hand it is a schematic/symbolic representation of a process; on the other, it represents elements within a realistic landscape. Within the landscape certain features are more 'realistically' depicted than others. The hill, sun, and water are presented fairly realistically but using familiar conventions (e.g. the sun is circular, with dotted rays coming out from it; the horizon is depicted by a straight line; the surface of the sea is indicated by lots of little wiggly lines; the fluffy clouds would look at home in a children's story book; rain is indicated by broken vertical lines); the dam is drawn schematically, but using 3D perspective to indicate its form; the power station is represented by a simple rectangle; the pylons are quite realistically 
drawn; and the current flowing between them is indicated by loose, broken, flowing lines. The result is a picture which has elements of a children's story book illustration, and elements of a more conventional scientific diagram, all mixed up together.

In the picture, a set of arrows following one another depicts a circular motion. The arrows do not actually form a closed circle and that helps one identify the point from which one should start the narrative. There seems to be an imaginary diagonal line from the bottom left to the top right (running alongside the slope of the mountain) which splits the image in two parts. In the top part of the image the arrows refer to changes to water, whereas in the bottom part the arrows refer to energy changes. In the top part, moreover, the arrows have multiple roles: they attempt to show the physical transfer of the water from the sea to form clouds, they indicate a sequence (by linking the various stages in the process, which are described with words), and finally, in some cases they also indicate direction (rising vapour, or falling rain). The arrows in the bottom part of the image seem to serve different functions as well. Whereas the arrow connecting the dam on top of the mountain to the power station some distance below can be thought of as showing the flow of water towards the power station, it is difficult to see what the arrows, running through the power station and coming out of it represent - the electrical current? One can easily mistake them as showing the flow of water downhill, especially because they run alongside some wavy lines which can be thought of as representing a river. Moreover, the arrows pointing downhill appear to show the direction of the electric current, but in fact they are misleading, as electricity does not in any real sense 'travel downhill'.

The use of the verbal elements (words and phrases) is also similarly ambiguous. In the upper part of the picture all mentions of (forms of) energy appear in italics, whereas mentions of water appear in simple small font and object labels, such as 'sun', 'sea' and 'power station', in simple capital font. So, under the word 'SUN' which lies on top of the schematic picture of the sun one can read 'Nuclear energy'; then one can read the word 'radiation' on top of the sun's rays again in italics, which could imply that this is another form of energy; and then the elliptical phrase 'Gains gravitational energy' under the arrow showing vapour forming into water drops. This last sentence obviously refers to water and would have been better placed under the arrow showing vapour rising. The phrases in the bottom part are different. Firstly they have a different syntactic structure; they do not now refer to changes but function as labels of the entities (real or abstract) the reader should be focusing on. So, it is not now 'water evaporates' or 'vapour rises' but 'water in dam' or 'energy as electric current'. Secondly, two out of the three talk about energy (as opposed to the ones in the upper part which mostly refer to water) and are clearly the dominant ones as they take most of the space of this bottom part of the image; the one which refers to both water and energy is confined in the top right position. Moreover, the 'energy' phrases in this part talk of 'energy of X' or of 'energy as $\mathrm{X}$ ' ('energy of movement' and 'energy as electric current') and not of ' $\mathrm{X}$ energy' ('gravitational energy' or 'nuclear energy') as before, and in this way seem to be telling the reader that the principal actor/entity to look for is 'energy', which is one and the same although found in different disguises.

Concerning the salience (or absence) of the various elements in the picture, we observed the following: In the picture, the dam and the power station are clearly intended to be salient. The dam, as already mentioned, appears shaded and drawn in 3D perspective, and the power station is labelled in large letters which make its label stand out from the other verbal elements. However, there appears to be a fair amount of information 'missing' from this section of the diagram which is likely to raise a number of questions in the mind of the reader: what actually happens in the dam, and in the power station; what is the precise role of the turbine; and where exactly does the current go after it leaves the power station? 
Moreover, the electricity cables seem to come to an end in mid-air; there is no indication of any destination for the electric current, other than visually the sea.

We take the aim of this picture to be to engage the pupil-reader to think about and identify the transfer of matter and energy in the depicted system. However, following the above discussion, we would suggest that the ambiguity and/or inconsistency of symbols and verbal elements in the document, in conjunction with the particular salience of the various elements in it, may encumber the attainment of this aim. Having said this, we need also to concede that some of these ambiguities may be accounted by the intention of the authors (Dobson 1987b). In particular, the distinction between movement of substance and transfer of energy seems to be acknowledged as conceptually very difficult for pupils to make, and hence as counter-productive for the teacher (and perhaps also the textbook) to make. In their own words:

'Pupils, of course, find it very difficult to distinguish the abstract notion "energy" from the "thing" associated with it (the moving wheel, light, steam, "electricity"). And they would need to learn a lot more about the "things" to be able to do so. Hence it is counter-productive to be too definite about what energy "is" at this stage".

This suggests that images from this source may be left ambiguous. Our purpose will be to see how pupils cope with any such ambiguity.

\section{Research questions}

The research questions for this document are:

- How does the combination of schematic and realistic representations in the document affect the meanings the pupils draw from it?

- How are the selection and highlighting of the represented elements interpreted and assessed by the pupils?

- How do the pupils deal with the ambiguity of symbols and/or verbal elements identified?

- How will the pupils extract an integrated meaning from the different elements (words, drawings, arrows) in the document?

\section{Pupils' difficulties with the document 'Hot on the Trail'}

Given the above research questions the categories which proved pertinent in analysing the data collected from the pupils in relation to this document are:

(i) Real world objects versus schematic or symbolic entities $(R / S)$

(ii) Selecting, highlighting or missing elements in a representation (SEL)

(iii) Similarity of symbols (SIM)

(iv) Integration of the reading of one or more representations (INT)

(v) Reading verbal elements (VE)

\section{(i) Real world objects versus schematic or symbolic entities $(R / S)$}

The children seem to be drawn to those parts of the picture which are more familiar to them. More children talk about the upper part of the diagram, involving natural elements, than the right side and lower areas, depicting modern technology. Comments on the upper part tend to be much more detailed than those on the right hand part. The lower section of the diagram appears to have been ignored by the majority; only three children mention the 'current' and only one of these mentions the pylons. Saira admits 'all that stuff I don't know about... I didn't really notice it... I guess I didn't look'. She also points out 'I wouldn't know that's a dam'; the schematic representation does not carry any meaning for her. Ruth, too, complains 'I find it difficult because the pictures are not drawn clearly'. 
Taking familiar 'real-life' features as a starting point, the children interpret the diagram in different ways. These are outlined below.

\section{Environmental features in relation to weather and water cycle}

The children appear to focus primarily on, and draw more meaning from, the real-life depiction of familiar environmental features in this diagram. Saira, for example, sees the picture as a whole outdoor scene: 'It's a picture of a hilly area near the sea'. Most of the children see the sun and water as particularly salient. Some think the picture is about the weather, and energy in connection with the sun.

Other children interpret the diagram as simply showing a water cycle. Mahmoud, for example, says:

'I first notice that the diagram is showing a water cycle. [...] I notice that the cycle starts off with the rain falling from the clouds and finishing at vapour forms into water drops. [...] The story behind this picture is that water can be very useful. It tells us that water is not wasted. It shows us how water takes each step. It shows us how it gets to the sea and how it becomes water vapour again'.

This interpretation focuses on the conversion of substances, not on what happens to the energy.

It is also thought by some of the pupils to show just the first part of the water cycle. Marcia, for example, thinks,

'The story behind this picture is a type of rainfall. The sun heats the sea (water) the water evaporates, the vapour rises, it then condenses in a cloud and then precipitation - meaning it rains. The rain then falls in a dam'.

Interestingly, Marcia stops at this point; she does not even attempt to understand the other representations. She explains further,

'To make sense of this picture you have to think about rainfall and you have to think of evaporation, condensation and precipitation'.

Others, on the other hand, start off interpreting the diagram in this way, but then see the other representations and realise that they need to rethink. Rajab explains, 'When I see the sun and the water and some clouds there, I just..., I got a feeling...', but 'them two (the dam and power station) were unexpected for me', they lead him to see that it is 'a bit of both, rain cycle and making electricity'.

\section{Landscape (variation in height) in relation to the lower half of the diagram}

The realistic hilly landscape setting can be seen as both helpful and distracting. The idea of height is important for an understanding of some aspects of the process, but not for others. It is important in relation to water rising from the sea; also the fact that it falls from the dam is crucial for an understanding of how the turbine works. But the landscape is not at all relevant for an understanding of how electricity flows through cables. Moreover, as explained previously, the depiction of the electric cables can be misleading.

In fact, five children experience difficulty with this section: they all think the lines represent water travelling downhill to the sea. (There are other problems with this particular part of the diagram, which are dealt with in the following sections.)

In conclusion, it seems that the drawings of natural features are in general more easily accessible to the children; some children are taking more meaning from the realistic elements than from the other features; and some of the realistic features may seriously mislead the reader.

(ii) Selecting, highlighting or missing elements in a representation (SEL) 
As discussed previously, the roles of the dam and the power station in the picture, though salient, do not seem at all obvious. Some examples of the children's understandings of these are outlined below.

\section{What happens in the dam?}

Habiba makes sense of the water in the dam as follows: 'it just goes down, so some of it is being used and dropped, some of it is just going on the floor and then it travels down to the sea'. She expresses frustration with the image: 'the picture is very poor... it doesn't explain it properly... there isn't energy or movement; it doesn't tell you it's dropping down... it doesn't say it clearly enough, that's not right'. Another pupil thinks that the water is formed into electricity while it is in the dam, but similarly complains: 'It doesn't show how the energy is formed here, the electricity'. He further suggests that there could be 'a separate picture next to it, like showing clearly how, how it goes into the dam and how it comes out again'.

\section{What happens inside the power station; what does the turbine do?}

Several children express confusion about the power station and turbine. A typical comment is: 'It's to do with the power station, I don't actually understand that'.

Some children skip briefly over this section of the diagram, whereas others attempt to make some sense of the information. Marcia suggests that electricity somehow helps the water to move: 'the electric currents help it flow'. Habiba, on the other hand, has a vague idea that the water is turned, but is very unsure about this: 'I think it's being turned by a turbine engine inside but I don't know'; for her the turbine seems to perform some kind of magical transformation: the water is (literally) 'turned into electricity'. For these children, water seems to be a passive substance which is acted upon in some way.

Finally, only two pupils seem to have a fairly clear understanding of what happens inside the power station. One of them, Rajab, describes it: 'The water drives the turbine which makes electricity energy as current flowing through pylons to people's houses'; '...making electricity with hydro-electric power'.

This part of the diagram is clearly not self-explanatory. The children are filling in the gaps in whatever way they can, some more successfully than others. For some of them it is too difficult even to attempt to understand. Mahmoud concludes: 'I think more information should be added to help understand'; 'It should have explained more from that stage to this stage' (i.e. the dam to the power station).

\section{What happens after the power station?}

There is some confusion as to what 'form' the energy takes, what it does, and where it goes, when it comes out of the power station.

Marcia and Habiba seem to think that water leaves the power station, but they are not sure where it goes from there. Marcia asks, 'I think it, the water goes right down, does it go down to the sea, or does it stay in? I'm not sure where it goes after that'. Habiba says 'it's been a bit built like a river... it to go down, but to go from the power station as a source of electricity'.

For Belinda and Fran water leaves the power station and goes back down to the sea. Mahmoud, on the other hand seems to think that electricity leaves the power station, and the current (or the wires containing the current -it's not quite clear what he means) ends up in the sea: 'energy as electric current goes... through the (Int: wires) ....wires, into the sea' but he also says 'It shows us how it gets to the sea and how it becomes water vapour again' suggesting that he may be thinking of the lines as representing both water and electric current. 
Rajab, as before, is the only child who indicates some understanding of the missing elements in the final part of the process: 'It goes as energy in electric, as electric current, to homes and power stations'.

Clearly this lower part of the diagram is very difficult to understand. One of the reasons could be the fact that there is no clear destination shown for the electric current. However, there are other possible reasons for the difficulties here, which are considered both in the previous and in the following sections.

\section{(iii) Similarity of symbols (SIM)}

\section{Problems associated with arrows}

One of the problems with this picture is that the arrows do not all mean the same thing, as discussed previously. This contributes to the difficulties, already mentioned in the previous two sections, that the pupils have in connection with the lower section of the diagram.

Similarly, the arrangement of the arrows so that they almost form a complete circle give rise to erroneous interpretations, as predicted; some children interpret the diagram as representing a cycle. Drawn to familiar environmental features (see corresponding section above) they tend to interpret the arrows as indicating movement of substance and may then interpret the whole document as a diagram of the 'water cycle'.

\section{Problems associated with flowing lines}

We also suggest that the similarity of the representation of the electric cables with that of the lower slopes of the hill - both are depicted as light, broken, flowing lines - may partly explain why some of the children (see sections above) interpret the electric current to be running downhill towards the sea. Furthermore, the fact that the lines representing the electric cables look very much like the kind of lines conventionally used to represent running water may also explain why some of the children think that they represent water flowing downhill.

\section{(iv) Integration of the reading of one or more representations (INT)}

In order to understand this diagram, the reader has to move through it, extracting meaning from the different elements (words, drawings, arrows) which are intended to work together to convey meaning. The reader has to decide in which direction to read, and how to read, the relationships between components. In one of the pupil's words, 'This is not a very complicated piece but you need to (?) read it in a special way'. We have suggested that it is actually quite deceptively complex.

\section{Problems associated with reading the diagram as a cyclical set of connected images}

The arrows indicate that the pictures and words are intended to be read clockwise, from left to right and round from right to left. Some children see it as a series of 'steps': Fran, for example, says: 'I can see the system which starts from the sea to energy as electric current. And all the steps which show it'. The idea of a cycle is helpful as a starting point, for getting a sense of direction in reading, as one of the pupils points out: 'You need to read the cycle going round clockwise'; he talks about going on 'a trip around the cycle'.

However, the idea of a cycle is not helpful, if adhered to too closely. Mahmoud says, 'To make sense of the picture you have to see where the cycle starts off. You have to follow the arrows and read each step until you get to the end of the cycle', but as Saira points out, a cycle has 'no starting or finishing point', so this analogy is misleading. There is a 'finishing point', in the sense that electricity ends up 
being used in all sorts of places (e.g. people's homes), but this 'end point' is not represented in the picture.

Marcia, faces a different kind of problem. Having interpreted the diagram as a depiction of the 'water cycle', she says, 'I find it difficult to make sense of why the power station and electric currents are in this picture'. The idea of a cycle restricts her interpretation.

\section{Problems associated with integration of visual and verbal elements}

Some children comment on the need to read the verbal information. Fran explains: 'You read the sentence written at the top of the sheet. Then start from the beginning then read each step and go along until you finish. Then you can see what is happening'.

However, this is not always straightforward. Habiba found the words in the area to the left of the clouds confusing: 'How can it just go up, if it's like in little drops?' Because the words 'vapour forms into water drops' are positioned to the left of the cloud, this suggests to her that the water is already in drops before it becomes a cloud.

Similarly, Matthew understands that the water vapour gains gravitational energy 'just before water vapour goes into drops', because the words 'gains gravitational energy' are positioned slightly to the left of 'vapour forms into water drops'. He found this quite difficult to understand; he says he 'had to think about what that means'.

Problems associated specifically with verbal information will be dealt with in the next section.

\section{Problems in interpreting individual visual elements and relationships between them}

The children faced particular difficulties in understanding the processes represented by the pictures of the dam, the power station and the electric cables, and the arrows which link the three. These are indicated in previous sections.

\section{(v) Reading verbal elements (VE)}

\section{Difficulties in understanding particular words and phrases}

In this document, the words carry a good deal of the meaning. Some children have difficulties because they do not understand particular words or phrases.

Fran says, 'I find it difficult when it says "water in dam (still with gravitational energy)"; I don't really get that'. Rajab too has problem with the word 'gravitational' and also with 'nuclear'. He says, "The "nuclear energy", "radiation" and "water in dam (still with gravitational energy)". Because... the word "nuclear" makes me confused. The "water in dam" also confuses me because I (doubt?) if the moon (drag?) the gravitational or if it's the Earth. Another word which causes confusion is "turbine".

Marcia says, 'I find it difficult to make sense of why the power station and electric currents are in this picture, although it says "Energy of movement in water and in turbine". She has written a question mark under 'turbine' and underlined it, indicating, we assume, that she does not understand the meaning of the word.

\section{Difficulties arising from insufficient written information}

For most children the diagram does not contain sufficient information about the process for them to fully understand the process depicted. Ruth says she found it difficult because, 'there are not enough words to describe it'.

\section{Summary}

Overall, this document seems to cause substantial difficulties to the pupils. 
- The presentation of schematic representations within a realistic landscape creates confusion; in addition some of the realistic features seem to mislead the reader.

- A fair amount of information is missing from several parts of the diagram and the words which refer to them are heavily loaded in meaning and content knowledge; as a result the children need to depend on their own understandings to make some sense of these parts.

- Finally, the use of arrows to represent different things and their arrangement, which implies a cyclical process, contribute further to the ambiguity and obstructs the intended reading of the document.

\section{Document 'Making fuels go further'}

\section{About the document 'Making fuels go further'}

This picture is found in the chapter on 'Energy', on the right page of the double-page-spread section called 'Running out of energy' and appears under the heading 'Making fuels go further' in a science textbook (Coles et al. 1989) for 12-year-old pupils. It is a quite complicated picture (see Appendix 3): It spreads over more than half of the page, it includes a variety of symbolic and realistic representations and there are very few linguistic clues about how one should go about reading it. It is a conceptual picture to be worked on. All its elements are drawn schematically at eye level and front on, on a neutral flat background. They represent generic categories of entities; each is very selective about what is relevant.

There are two horizontal lines which split the picture in three parts or levels. Each level relates to the one below with the aid of thick arrows. There is a weak narrative spanning the whole of the picture, but there are strong analytical and classificatory aspects in it. In general, one might say that from the top to the bottom one moves from the more general (whole) to the more specific (parts), or from the 'given' to the 'new'. Each level seems to have a different organization and thus different features prevail. On the first (top) level one finds the schematic pictures of an oil rig and of a crane on top of a coal mine. From these two pictures, which the reader is invited to consider as representing one thing (hence one arrow splits in three), three thick arrows lead to the pictures of a factory, a car and a house in the second level. The sentence immediately preceding the picture explains what these arrows represent. It says: 'Almost all our energy goes on our homes, transport and industry'. Above each of these three pictures one can read the generic name of what they stand for, that is, 'industry', 'transport' and 'homes' and a percentage showing how much energy goes to each. The pictures of the first and second levels have a whole-parts relation, thus their combination is analytical descriptive. This is further testified by the fact that the percentages of the three parts add up to $100 \%$.

Now from each of these objects-categories two examples are given vertically, one below the other in the third part/level of the picture. An arrow invites the reader to examine each category and its examples separately. Here the arrows do not show transfer of any sort, but simply link the superordinate category with its examples. A 'kind-of' classificational relation links the second with the third levels. All participants in the third level are distributed symmetrically across the picture space; vertically in each of the three categories one moves from the more efficient to the less efficient, and in two cases also from the larger to the smaller; horizontally, the analytic relation introduced in the second level is restated. Efficiency is implied, since next or above each of the schematic pictures showing for example a large and a small power station there is a bar chart showing how much of the energy is provided as electricity and how much as heat and sound. A large power station is thus more efficient than a small power station, a diesel engine more efficient than a petrol engine and a fluorescent light more efficient than a normal light bulb. The way the bar charts 
are drawn invites the reader to compare for each example how much of the energy ends up as heat compared to as other forms.

Overall, the individual components seem to be weighted equally, in terms of the amount of space taken up, although the items at the top are presented rather more substantially, with heavier shading. This could be misleading, as the most important part of the message seems to be carried by the images and figures in the lower half, and particularly below the second set of arrows. The arrows themselves are highlighted through shading and size, and clearly intended to be salient. The figures indicating the amount of energy used by industry, transport and homes are highlighted in bold; this could suggest that they are more important than the figures lower down, although we do not necessarily think this is intended. The bars showing 'wasted' energy are shaded, but none of the related figures are highlighted in any way.

The aims of the section in the textbook where the picture is situated are identified in the associated teacher's guide (Coles et al. 1988) by reference to the relevant National Curriculum (DFE 1995) recommendations for the teaching of energy. These latter specify that pupils should:

- explore the generation of electricity from different energy sources;

- survey national and global sources of energy;

- be introduced to the idea of energy efficiency.

We take the main aim of this picture in particular to be the last one, i.e. to introduce the idea of energy efficiency to pupils. In order to understand the intended message, pupils are expected to follow the arrows and focus on the 'new' information at the bottom of the page, specifically the different figures associated with wastage, and to understand the comparison being made: that some items are more efficient than others. In other words, they are expected to by-pass the previous upper section fairly quickly and concentrate on the lower half. We suggest that this may not be so straightforward; the particular highlighting of the upper part may distract pupils and lead them to unhelpful interpretations.

To conclude, this picture undoubtedly requires hard work on the part of the reader. It contains a mixture of representations, even knowledge representations such as bar charts; it is organized differently at different levels; in it arrows serve different functions. How will the pupils cope with all these features?

\section{Research questions}

The research questions for this document are:

- What do the pupils see as the main message of this document?

- Does the fact that the document contains a mixture of representations, from realistic to knowledge representations, give rise to any difficulties in the reading of the document?

- How do the pupils extract an integrated meaning from the different representations?

- How do the existing highlighting of particular elements and the mixing of different compositional structures in the document affect the meanings the pupils draw from it?

- How do the pupils deal with the fact that the arrows are assigned different roles in different parts of the document?

\section{Pupils' difficulties with the document 'Making fuels go further'}

Given the above research questions the categories which proved pertinent in analysing the data collected from the pupils in relation to this document are: 
(i) Reading images through theoretical lenses

(ii) Real-world versus schematic or symbolic entities $(R / S)$

(iii) Selecting, highlighting or missing elements in a representation (SEL)

(iv) Reading of compositional structures (CS)

(v) Similarity of symbols (SIM)

(vi) Reading verbal elements (VE)

(vii) Integration of the reading of one or more representations (INT)

\section{(i) Reading images through theoretical lenses}

The children's responses indicate that they are bringing a range of ideas with them which contribute to their interpretations, but not always in a helpful way. Saira, Belinda and Habiba provide some interesting examples.

Saira says:

'The story behind this picture is the way fuels are used and where they are used. It also shows how one fuel can be used to release more than one type of energy'.

This interpretation, focusing on production of different kinds of energy, rather than the wastage of an 'unwanted' type of energy, may have its roots in earlier classroom work. It may be partially explained by another comment, made by the same child, indicating association of particular images with particular scientific content and showing a connection in her mind with other work that has been done in school:

'There are pictures of things I always see in science text books. I see these pictures on the sections about energy and transfer of energy'.

For Belinda the idea of energy waste is associated with what humans can do in the home to prevent wastage, rather than with how different items 'waste' different amounts of energy. For her the main message is

'That we use too much electricity when we could cut down instead of wasting it, by turning things off... It helps you understand why you should cut down in using things as it uses a lot of electricity'.

This information is not illustrated in the diagram; the child is clearly drawing on her own previous knowledge about fuel wastage. She does recognise that the picture is about energy efficiency, and the idea of some things being more efficient than others, and suggests: 'you could cut down on using fuel and things like that by using, by compromising using different things' but her responses to the examples indicate some confusion: 'I'm not sure... that you can cut down'. She understands the lower set of examples to show 'different results' to the examples above, but has difficulty explaining their significance. The problem seems to stem from a general focus on use rather than transfer of energy.

Belinda and Habiba both seem to be approaching the document with the idea that energy is used as well as 'produced' by the items in the lower half, and interpreting the 'wanted form' of energy in the first two pictures as representing use rather than transfer. Belinda notes 'the diesel engine used like a lot more movement and in the petrol engine it only had 25\% movement'; her response seems to indicate that she thinks the petrol engine is more efficient because it 'uses' rather than 'produces' less movement. When trying to understand the smaller power station Habiba says 'the less electricity is used but still the more heat and sound is wasted', and when focusing on the car she says 'the less it uses, it wastes more'. She finds this confusing since she is bringing with her the understanding that using electricity on a large scale is generally more economical than on a smaller scale: 'if you use large quantities, the less is wasted'. However, she finds this theory of 'bigger is better' more helpful in relation to the picture of the lights, explaining: 'if you had a bigger house and light the whole house you'd still waste less than someone with a smaller house and like four light bulbs in the whole house'. 
Belinda also interprets the examples based on the idea that one form of energy 'produces' another:

'I think it was just showing that other things which we use in the industry and in the transport and homes, like electricity, the heat and sound that came from that, and with transport, the movement and the heat that came from the movement, and in the homes, the light and heat that came from the light, so heat was coming from the electricity, the movement and the light'.

She has the idea of a 'by-product' form of energy and thinks it is 'produced' out of the first ('wanted') form rather than simultaneously alongside it.

\section{(ii) Real-world versus schematic or symbolic entities $(R / S)$}

This does not seem to be a major cause of difficulty, but we found two comments interesting. For one child the schematic images at the top seem to carry more specific meaning, being associated specifically with nuclear power stations; Ruth says, 'The story behind the picture is nuclear power stations and things like that'. In this case, though, the under-generalising does not seem to adversely effect the child's understanding of the main points.

For Belinda, the more familiar 'realistic' images on the lower right side seem more salient than the more schematic ones on the left. In talking about the items on the right, the house and the lightbulb, which clearly relate to use of energy in everyday life in the home, she appears much more confident than when trying to explain the information about power stations. In this case, associations with the familiar items on the right, and lack of familiarity with the items on the left, may both be contributing to her difficulties, in different ways.

\section{(iii) Selecting, highlighting or missing elements in a representation (SEL)}

\section{Highlighting}

As discussed earlier, in order to understand the message, the reader is expected to follow the arrows and focus on the 'new' information at the bottom of the page. However, this is not what the children tend to do. The children do choose to focus on specific parts of the diagram and ignore or deal very briefly with others, but interestingly, they do not all choose to focus on the same parts, and some of them appear to pay little attention to the lower set of examples.

We would suggest that the large shaded arrows may draw attention to themselves in a way which could lead to an unhelpful interpretation. For several children they are an important element which leads them to interpret the 'story' largely as the 'journey' of fuel from its source to use as different 'forms' of energy. For example, Matthew says, 'The story behind this picture is in a sort of flow chart and shows where and how fuel is used'. Although wastage may be mentioned, for these children it seems that the 'life' of the fuel itself, and its 'journey' represented by the arrows, may be equally, if not more salient.

Some of the percentage figures are written in bold. Several children express confusion in relation to the meaning of the figures in bold. For example, in Fran's view 'The story is that everything that uses energy wastes it, i.e. industry $37 \%$, homes $31 \%$, transport $32 \%$ of energy wasted'. This response suggests that the child may be interpreting the figures in bold as referring to wastage.

The lack of clear highlighting of elements, especially in the lower half, may be why some children focus briefly on all sections: for them the fuels themselves, the places where they are used, the things that they are used for, and how much is used are all salient features. Ali says: 
'I have noticed that it shows how we get our electricity, gas and oil, coal, for transport, homes and industry. And the use of these substances through light bulbs, cars (engine) and homes... [...] The story behind this picture is where the energy goes after extracted and the usage of the substances as well as the percentage of the usage'.

In this case there is no mention of energy efficiency. Some children mention wastage, but briefly, in passing, rather than as the main focus. For example, Mahmoud explains:

'The first thing I notice about the picture on it is that the diagrams are about fuels. They are used in many things. They are important to us. It tells us how much fuel is used by different things.... Fuel is very important. By using fuel it is helpful but at the same time we waste important energy, at the same time'.

Some children focus largely on the middle and lower sections, but may not fully explain the information in the lower section. They appear to be following the arrows as expected but not moving further beyond them to the lower section. They understand that the 'story' is about energy wastage, things that use energy, and their varying efficiency, but they do not mention a distinction between the efficiency of specific types of things (e.g. different types of cars). For example, Simon says 'The first thing I noticed about the picture is a picture story of how energy is produced, used and wasted. I noticed that it showed how the energy was used and how efficient some energyusing things are'. Habiba says, 'It shows percentages of how much energy goes to different things like cars, industries and homes, it also shows how much energy is wasted'. The idea of wastage is still expressed fairly vaguely; these responses do not indicate a clear understanding of what the pictures and figures represent.

The child who appears to express the clearest understanding of what is represented on this page does not mention the fuel source at the top but moves directly to the parts below the first set of arrows, and focuses mainly on the images in the lower part of the page (the 'new' information). Rajab says:

'I notice certain types of industry, cars and homes. Recorded in percentages are the amount they are using for what they are supposed to do and what not, e.g. a car with diesel engine makes $60 \%$ heat which we don't want and makes $44 \%$ movement which we want. However, it is making less of what we want than what we don't want, so the car is inefficient. [...] Every picture tells a story about how energy is getting used up. It is saying that everything that uses energy also wastes it!! It is trying to say that efficient things use energy better and waste less. The picture tells us that most of our environment is making less of what we want than what we don't want'.

Rajab is making a clear distinction between 'given' and 'new' information in a way that other children are not.

\section{(iv) Reading of compositional structures $(C / S)$}

The layout seems to place demands on the children; the mixing of horizontal and vertical layouts, and correspondingly of analytical and classificational structures may be responsible for some of the confusion. (This needs to be seen in combination with the use of arrows - see also section 'similarity of symbols' below). Marcia explains 'At first it was a bit confusing (the way it is set out) but when you look at it a bit more you can understand it'.

The top-down (analytical) hierarchy in the upper part, with arrows pointing downwards between the levels, leads the reader to expect this hierarchical meaning to continue in the lower half. However, the items positioned one on top of the other at the bottom actually represent alternative possibilities (classificational structure). This is unexpected; they are not laid out side by side, as one would expect, with two diagonal arrows leading down towards them. Instead, they are arranged one on top of the other, as if in a vertical arrangement, with arrows from left to right.

This mixing of horizontal and vertical layout patterns, resulting in an unclear relationship between the main categories and subcategories, may be contributing towards difficulty in 
interpretation as expressed in those vague responses where the distinction between items of greater and lesser efficiency does not appear to have been fully understood.

\section{(v) Similarity of symbols (SIM)}

Ali says 'The arrow is difficult to read from the actual pictures'. It is not clear which arrow he is referring to, but either way the comment is not surprising, since the two sets of arrows carry different meanings. The second set of arrows does not represent a clear continuation in the 'flow' of energy. Each of the lower arrows leads to two items one above the other, which represent a choice of possible outlets, but with only the one arrow, in combination with the hierarchical layout (see section on 'reading of compositional structures' above), the fact that these are alternatives is not made clear.

The following interesting comment points to the lack of clarity resulting from the problems of 'reading of compositional structures' and 'similarity of symbols'. Ruth says, 'The story behind the picture is nuclear power stations and things like that produce energy which then goes to three main different places industry, transport, homes. It then separates again and they are showing so much of the energy is wasted on heat and sound which is not needed'. In fact, the arrows do not 'separate'. It seems to us that Ruth may be trying to make sense of a visual arrangement which does not communicate a very clear meaning, by applying her understanding of a form of visual representation with which she is familiar (i.e. alternative outcomes represented by separate arrows).

\section{(vi) Reading verbal elements (VE)}

There seem to be two problems in relation specifically to verbal information. Firstly, there seems to be a problem for some children in making connections between the words top left and the visual information and percentage figures, at the bottom. This demands a lot of effort; as Ruth says, 'I notice the picture is explaining how fuels go further (guess from the title). I think it looks quite complicated and the picture does not tell me enough for me to understand it properly. Also it looks a bit boring'. The figures and bar charts may in themselves appear helpful, but without further explanation, may not always communicate their full meaning to the children. Ali says 'The picture helps a bit by giving the percentages, but the rest it doesn't exactly tell you what it is about'.

As indicated above (under 'highlighting'), some children do not actually mention wastage. This may be partly due to the fact that the idea of wastage is not made explicit, verbally, in close proximity to the percentages.

On the other hand, some children appear to over-generalise from the information in the paragraph and assume that all the percentages given below indicate wastage. Fran, for example, says

'I read the headline so I can see that it's about fuels and to do with going further. I read the paragraph which is stating that everything that uses fuel also wastes it. I look at the picture, I can see percentages of amounts of energy waste. [...] The story is that everything that uses energy wastes it, i.e. industry $37 \%$, homes $31 \%$, transport $32 \%$ of energy wasted. Another example would be a light bulb, it uses energy to make light. It gets hot which is a waste of energy'.

Here the figures in the upper section are seen as representing waste, and the examples below as a parallel set of examples of wastage, not as representing percentages of the amounts shown above. Clearly, the interpretation of the figures needs to be seen in relation to features such as highlighting and layout (discussed in previous sections). 


\section{(vii) Integration of the reading of one or more representations (INT)}

The relationships between images and percentages in the lower half of the page, and their relationship to the written information top left, seem to have caused particular problems. Issues discussed in the four previous sections ('SEL', 'CS', 'SIM', 'VE') are relevant here.

\section{Summary}

For most children, it is the lower part of the diagram (the information concerning efficiency, represented by drawings and figures) which tends to cause problems. The children appear to be experiencing difficulty in dealing with the 'new' information. This suggests that there may be problems with the way in which the information here is presented.

- As indicated above, difficulties could be partly due to highlighting and relative equality in terms of representation of items selected to be depicted in different parts of the diagram, and from missing information, in relation to the images and percentages in the lower half.

- They could also be due to the mixing of horizontal and vertical layout patterns, and to the ambiguous use of arrows, which mislead and result in confusion.

- Finally, the required integration of readings of visual, verbal and numeric information puts a considerable demand on the reader, who may as a result end up relying on superficial and/or familiar associations to make sense of the document.

\section{Concluding remarks}

In this paper we have looked at pupils' readings of three documents on 'energy' taken from popular science textbooks, with the aim of describing the possible difficulties the documents might have presented to the pupils. In doing this, we feel we have made a strong case that the 'reading' of pictorial representations is not at all trivial or straightforward. This is even more the case when the representations are of conceptually difficult ideas, and are intended to be used as teaching/learning aids. As a consequence, we believe that the issues which can be seen as pertinent in understanding better the difficulties involved in this reading merit indepth investigation.

More particularly, our investigation has raised some issues, which we believe are worth stating.

All documents discussed and analysed come from respected and widely used books, which share a strong commitment to visual communication of scientific ideas. They are therefore not in the least 'hard cases', chosen to exhibit problems; other texts can be expected to show a similar range of difficulties. The results therefore suggest that more attention needs to be paid to the construction of such images, if they are to function more effectively.

The results also show that pupils do in fact work to understand the images. Their difficulties are not simply ones arising from inattention, or only from the fact that they necessarily 'read' information through their own 'theoretical lenses'. This indicates that teachers need to spend time and effort talking through the meaning of the images, and false meanings they may convey, with pupils.

It is too often supposed that images are 'transparent' - that they yield their meaning directly and simply. The analytical tools we have found it useful to employ suggest that this is not at all the case - that quite complex and subtle organizations of elements structure the meaning of images. 
In more detail, we began with a list of textual/graphical features of images, which might present difficulties to pupils. We have been able to identify examples of each type of feature listed, giving evidence of the difficulties they do actually appear to create. The results suggest that the list is a good starting point for a critical examination of the kinds of images one may find in science textbooks. Converting the list into a form of advice to authors, we would say:

- be careful when mixing symbolic and real entities;

- pay attention to highlighting of elements intended and accidental;

- encode different meanings of similar symbols in different ways;

- pay attention to the wording and placing of verbal elements;

- careful layout is needed when several images are to be integrated in one;

- remember that compositional structures are used to read meaning into images, even when this is not fully conscious.

Finally, we think we have shown that each of the images could have been improved, if the authors had had access to appropriate data from pupils' responses, analysed in the above or similar terms.

\section{Acknowledgements}

We would like to thank the pupils and teachers of Gladesmore Community School, King's Manor School and South Camden Community School who participated in the above research.

\section{References}

Coles, M., Gott, R., Price, G., \& Thornley, T. (1988) 8. Energy. In Active Science 2 Teacher's Guide (London: Collins Educational), 93-101.

Coles, M., Gott, R., \& Thornley, T. (1989) 8. Energy. In Active Science 2 (London: Collins Educational), 41.

DFE (1995) Science in the National Curriculum (London: HMSO).

Dobson, K. (1987a) 5. Energy. In Co-ordinated Science (13-16). Introductory Book - GCSE (13 yrs old) The Suffolk Development (London: Collins Educational), 54.

Dobson. K. (1987b) Teaching for active learning. Co-ordinated Science. Teachers' book. (London: Collins Educational).

Ellse, M. (ed.) (1993) Nuffield Science for Key Stage 3. Science Year 8. Teacher's guide (Harlow: Longman Group UK Ltd), 31-48.

Kress, G. and van Leeuwen, T. (1996) Reading Images: The Grammar of Visual Design (London: Routledge).

NUFFIELD (1987) 1. Energy. In M. Ellse (ed.), Nuffield Science for Key Stage 3. Science Year 8 (Harlow: Longman Group UK Ltd), 1.

Watts, D. M. (1983) Some alternative views of energy. Physics Education, 18, 5, 213-217. 


\section{Appendix 1}

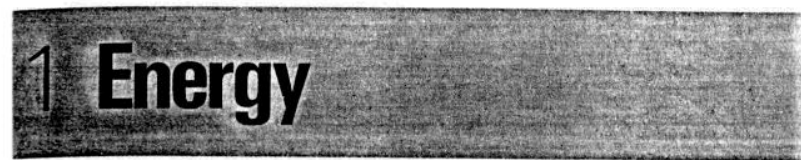

Have you ever head someone say: 'We live in a technological society"? Do you know what it means? When people talk about 'our society" they mean all the millions of people who live in our part of the world, say, in the United Kingdom. These people. including you and me, have many things in common. For example, the way we live depends on things which technologists have designed and made.

You can see a collection of such things on this page. The list concentrates on those which we use to move ourselves and our goods from place to place. You could say that they are all "technological forms of transport".

How many different forms of transport can you see in the pictures? Can you think of any others? Can you work out what all these forms of transport need to make them go"?
Picture 1

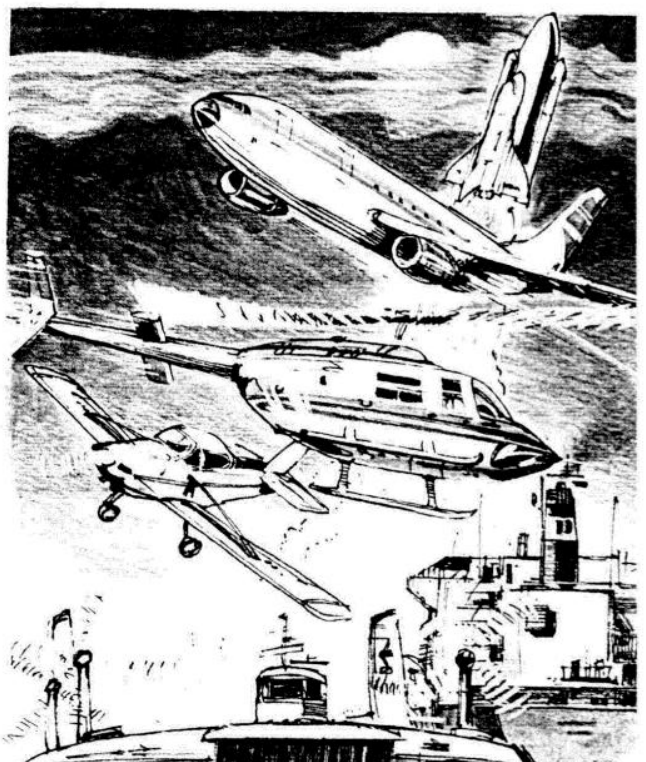

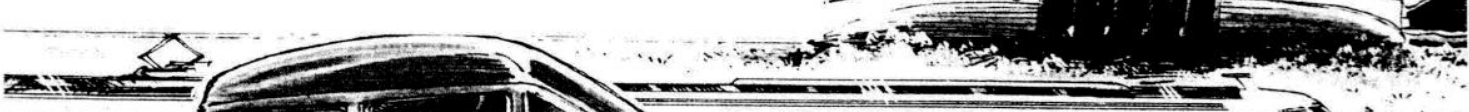
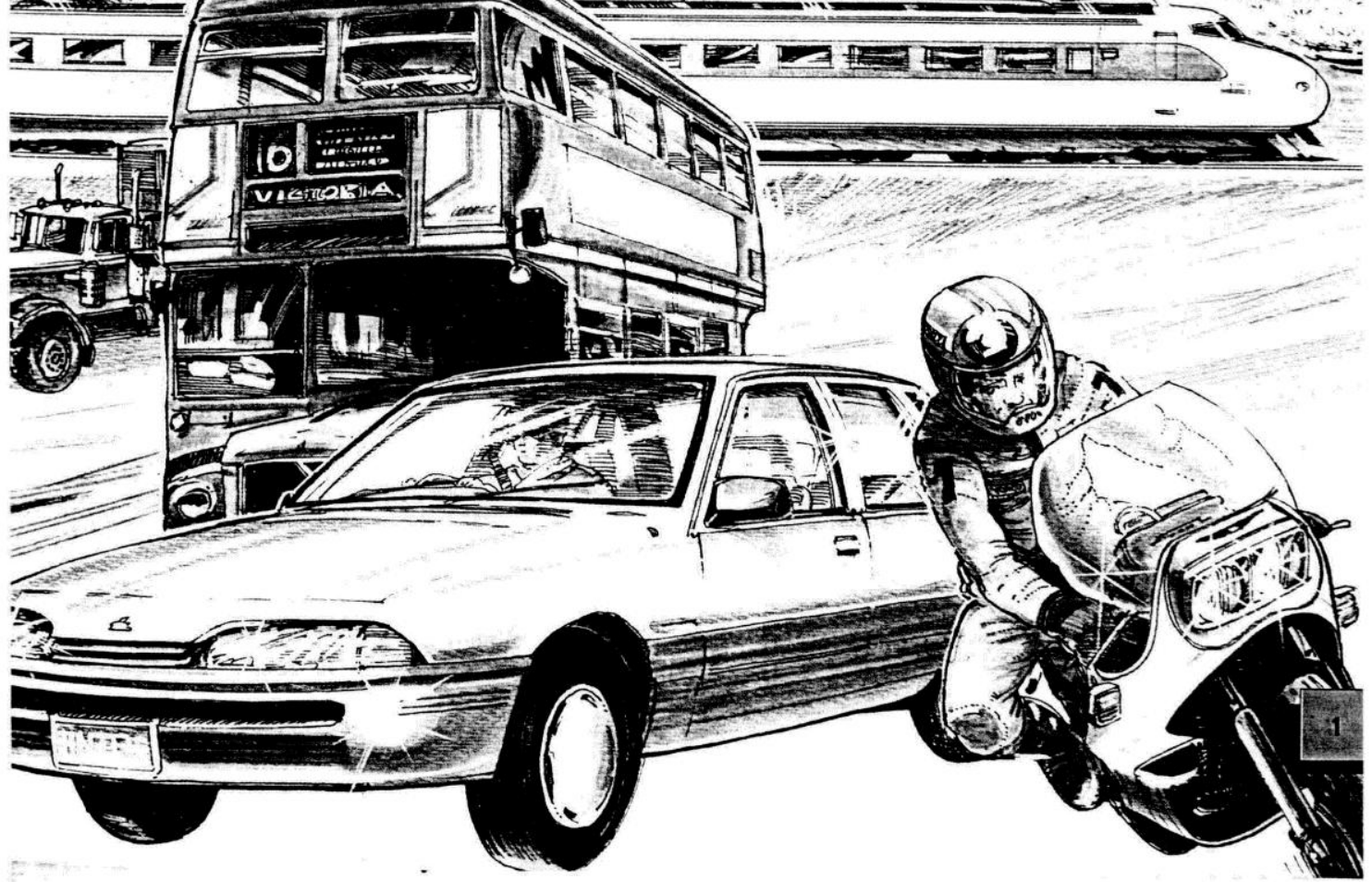


\section{Appendix 2}

\section{HOT ON THE TRAIL}

Tracing energy through a complicated system can be quite interesting.

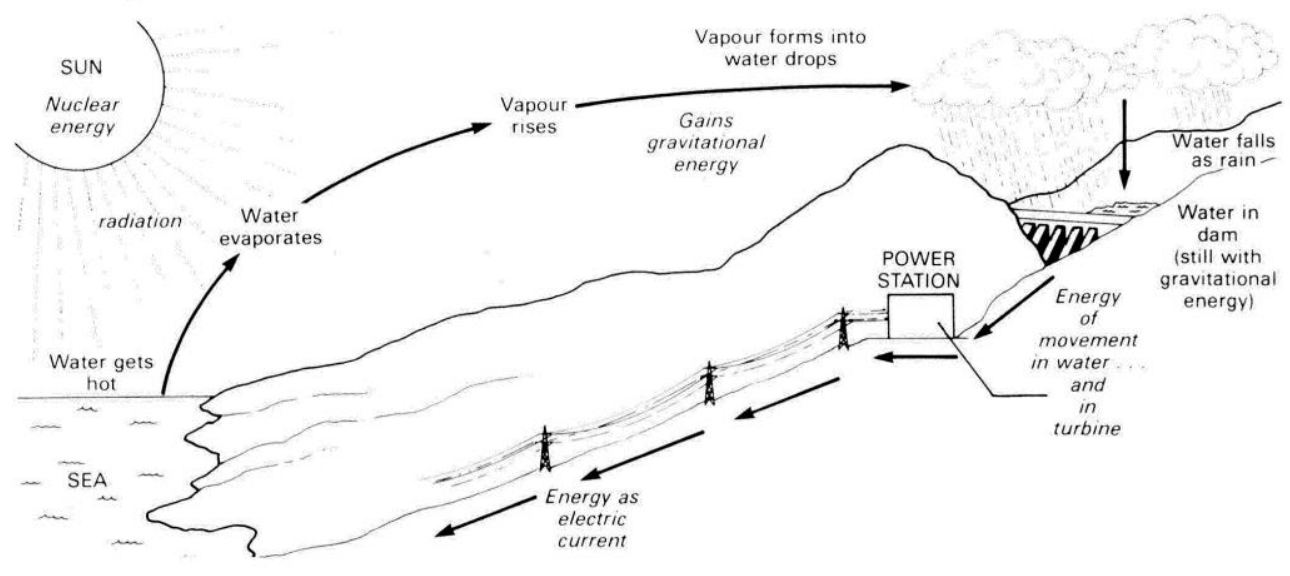




\section{Appendix 3}

\section{Making fuels go further}

Everything that uses energy also wastes it. For example, a light bulb uses energy to make light. At the same time, it gets hot, which is a waste of energy. Efficient things use energy better and waste less.

Almost all our energy goes on our homes, transport and industry.

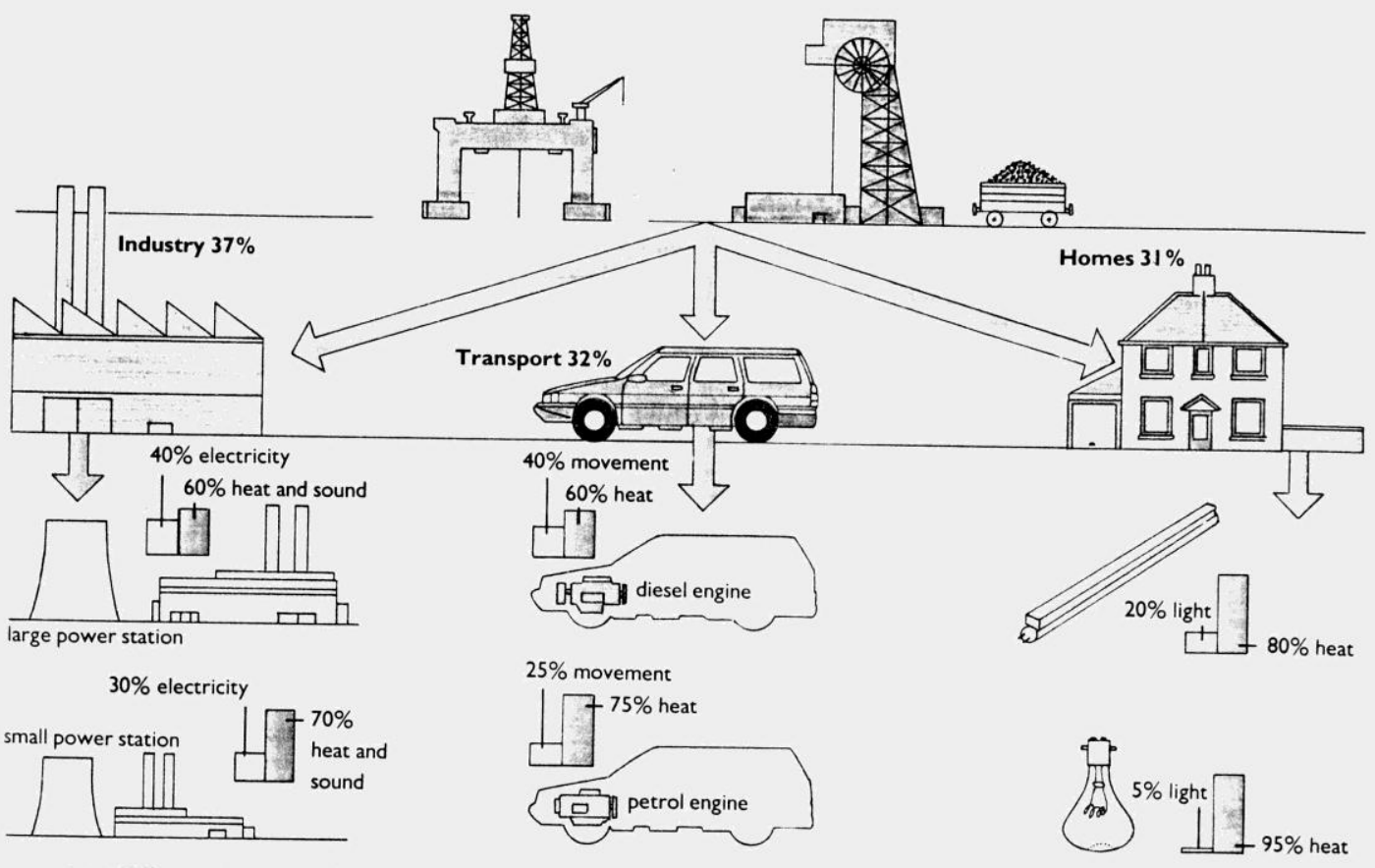

- Do a pie chart that shows where most of our energy is used.

\section{Cutting out waste}

- Give some examples from this chart of energy being used more efficiently.

An average British family uses energy like this:

Heating the house $\quad 40 \%$

Transport 25\%

Electrical goods $\quad 16 \%$

$\begin{array}{lr}\text { Heating water } & 10 \% \\ \text { Food eaten } & 5 \% \\ \text { Cooking } & 4 \%\end{array}$

Does your school waste energy?

- Plan and carry out a survey of energy wastage in your school.

- Write a report for your headteacher with ideas for improvements.

- Do a display that shows how a family uses energy.

6 Where and how do you think you could save energy?

\section{EXTRAS}

1 Do an energy wastage survey at home. Suggest to your parents that they should pay you a percentage of any savings!
Are you most efficient when you use energy quickly or slowly? Design and carry out an investigation with a bicycle. You can use ten complete turns of the pedals. Does the bike go further if you pedal quickly or $\$$ slowly? Think of some ways of getting
energy that will not run out. 